\title{
Markov Chain Monte Carlo Effective Range Analysis of Low-Energy Electron Elastic Scattering from Xenon
}

\author{
Kamil Fedus ${ }^{1}$
}

Received: 30 September 2015 / Published online: 9 November 2015

(C) The Author(s) 2015. This article is published with open access at Springerlink.com

\begin{abstract}
Modified effective range theory formulated as a Bayesian statistical model through the combination with Markov Chain Monte Carlo integration and fitting techniques is used to check the compatibility of different $\mathrm{e}^{-}-\mathrm{Xe}$ scattering data such as the total cross-sections, the momentum transfer cross-sections, and the differential cross-sections that were determined experimentally in the region of Ramsauer-Townsend minimum. On the basis of this predictive approach, the most probable value of the scattering length, $(-6.51 \pm 0.05) a_{0}$, is proposed. The present analysis suggests that the non-relativistic spinless effective range theory is suitable for the description of angular and energy dependencies of $\mathrm{e}^{-}-\mathrm{Xe}$ elastic scattering cross-sections below the threshold for first inelastic process.
\end{abstract}

Keywords Electron elastic scattering $\cdot$ Xenon $\cdot$ Modified effective range theory

\section{Introduction}

The modified effective range theory (MERT) for electron and positron scattering, originally proposed by O'Malley et al. [1], is frequently used to extrapolate measured cross sections down to zero energy, the region hardly accessible experimentally. However, the applicability of the original approach is limited to the very low energies (below $1 \mathrm{eV}$ in

Kamil Fedus

kamil@fizyka.umk.pl

1 Institute of Physics, Faculty of Physics, Astronomy and Informatics, Nicolaus Copernicus University, Gagarina 5, 87-100 Torun, Poland noble gases as shown by Buckman and Mitroy [2]) where rare experimental data are subject to large errors making the MERT extrapolation unreliable.

To overcome this problem, Idziaszek and Karwasz proposed [3, 4] a different approach to MERT: the expression describing the scattering phase shifts of angular momentum partial waves as a function of incident electron energy is obtained exactly using Mathieu's functions, i.e., analytical solutions of the radial Schrödinger equation with an adiabatic long-range dipole polarization potential $\left(\sim r^{-4}\right)$. The effective range approximations are introduced into the analytical solution exclusively for a short-range part of the interaction potential. We showed in a series of papers that such approach allows to extend the applicability of MERT almost to the threshold for the first inelastic process in many simple targets, including $\mathrm{He}, \mathrm{Ar}$ and $\mathrm{H}_{2}$ [5], $\mathrm{Ne}$ [6], $\mathrm{Kr}$ [7], and $\mathrm{CH}_{4}$ [8].

One disadvantage of MERT analysis of scattering data is related to multiparameter nature of the effective range expansions (approximating the energy dependence of shortrange effects). The multiterm fit to experimental data is always characterized by a certain portion of ambiguity since a large number of independent parameters can sometimes prevent their unique determination. Therefore, recently [9], we proposed to combine MERT with Markov Chain Monte Carlo (MCMC) fitting procedures (see [10]) in order to give the model a more statistical nature (in the terms of Bayesian statistics [10]) and to determine the uncertainties related to the fitting parameters.

MERT has been extensively used to analyze different cross-sections for all noble gasses; however, it has been rarely applied for Xenon atom. To the best of our knowledge, only O'Malley [11], Guskov et al. [12], Weyhreter et al. [13], and Kurokawa et al. [14, 15] used the original approach to MERT in order to extrapolate some low-energy 
$\mathrm{e}^{-}-\mathrm{Xe}$ experimental data down to zero energy. However, except the last paper, these analysis were done long time ago, when few datasets were available. Generally, it is expected that an important source of uncertainty in MERT analysis of Xe cross-sections will come from neglecting spin-orbit interactions which cause the phase shifts for $l>0$ to be different for the different spin orientations of the incident electron. Elaborated relativistic calculations [16-19] including spin-orbit interactions show that the phase-shift splitting due to the spin polarization is indeed noticeable for $\mathrm{e}^{-}-$Xe collisions; however, this effect is energy dependent and it is not very important in low-energy range $(E<$ $10 \mathrm{eV}$ ), particularly in the region of Ramsauer-Townsend minimum.

This work shows that MERT model is suitable for the description of low-energy $\mathrm{e}^{-}-\mathrm{Xe}$ scattering data even if spin-orbit interaction is neglected. In particular, it is proved that both the angular and the energy dependencies of scattering cross-sections can be well modeled within the framework of this non-relativistic model if the correct value of the static dipole polarizability is used an input parameter. The latter quantity intrinsically contains the contribution of relativistic effects which are necessary in order to theoretically describe such a heavy atom as Xenon. To further validate the present approach, MCMC-MERT is employed to check the compatibility of total cross-sections (TCSs) measured in the region of Ramsauer-Townsed minimum with experimentally determined differential and momentum transfer cross-sections (DCSs and MTCSs, respectively) available for the same energy range. On the basis of this predictive analysis, the most probable value of the scattering length and the effective range parameters are proposed. Finally, MERT-derived dependence of the $s$-wave electron scattering length for rare-gas atoms on the dipole polarizability is discussed.

\section{MCMC-MERT}

A semi-analytical approach to MERT, originally introduced by O'Malley et al. [1], has been discussed in details in our previous papers [3-5]. The combination of MERT with Markov Chain Monte Carlo fitting techniques (MCMCMERT) was introduced in ref. [9]. Therefore, only a brief description will be given here.

MERT analytical expression for partial wave scattering phase-shift induced by the spherical part of the longrange dipole polarization potential is given by the following expression (in atomic units) [1]:

$\tan \eta_{l}=\frac{m_{l}^{2}-\tan \delta_{l}^{2}+\tilde{B}_{l} \tan \delta_{l}\left(m_{l}^{2}-1\right)}{\tan \delta_{l}\left(1-m_{l}^{2}\right)+\tilde{B}_{l}\left(1-m_{l}^{2} \tan ^{2} \delta_{l}\right)}$, where $l$ is the angular momentum quantum number and $\delta_{l}=\frac{\pi}{2}\left(v_{l}-l-\frac{1}{2}\right)$. Here, $m_{l}$ and $v_{l}$ denote the energydependent parameters which have to be determined numerically for each partial wave using the procedures described in refs. [3-5]. Parameter $\tilde{B}_{l}$ is related to the additional phase shift that is induced by the unknown short-range potential (static, exchange, and short-range correlation interactions). This parameter is approximated by the quadratic effective range expansion [3-5]:

$\tilde{B}_{l}=B_{l}(0)+\frac{1}{2} R^{*} R_{l} k^{2}+\ldots$,

where $k$ is a wavenumber, $B_{l}$ is the zero energy contribution, $R_{l}$ can be interpreted as the effective range for a given partial wave, and $R^{*}=\alpha^{1 / 2}$ with $\alpha$ being the dipole polarizability. In the particular case of $l=0, B_{0}$ can be expressed in terms of $A$, the $s$-wave scattering length, as $B_{0}=-R^{*} / A$.

Using this semi-analytical model, we verified below that in the regime of energies for elastic $\mathrm{e}^{-}+\mathrm{Xe}$ scattering $(<10 \mathrm{eV})$, the leading contributions come from $s, p$, and $d$ partial waves $(l=0,1,2)$ while the contributions of higher partial waves are small and they are not modified by the short-ranges forces due to very high centrifugal barriers associated with large $l$ numbers. Therefore, the phase shifts for $3 \leq l \leq 100$ partial waves have been estimated using Eqs. 12-14 from paper by Ali and Fraser [20]:

$\tan \eta_{l}=k^{2} \alpha a_{l}+k^{4}\left(\alpha^{2} b_{l}+\beta c_{l}\right)$, for $l \geq 3$,

where $a_{l}, b_{l}$ and $c_{l}$ are given by

$$
\begin{aligned}
a_{l} & =\frac{\pi}{(2 l+3)(2 l+1)(2 l-1)}, \\
b_{l} & =\frac{\pi\left[15\left(2 l+1^{4}\right)-140(2 l+1)^{2}+128\right]}{[(2 l+3)(2 l+1)(2 l-1)]^{3}(2 l+5)(2 l-3)}, \\
c_{l} & =\frac{3 \pi}{(2 l+5)(2 l+5)(2 l+1)(2 l-1)(2 l-3)} .
\end{aligned}
$$

These analytical expressions describes the energy dependencies of elastic scattering phase shifts that arise from pure long-range inverse power forces including spherical dipole $(\alpha)$ and quadrupole $(\beta)$ polarizabilities.

Integral elastic $\left(\sigma_{I E}\right)$, momentum transfer $\left(\sigma_{M T}\right)$, and differential elastic $\left(\frac{d \sigma}{d \omega}\right)$ cross sections are calculated using the standard partial wave expansions:

$$
\begin{aligned}
& \sigma_{I E}=\frac{4 \pi}{k^{2}} \sum_{l=0}^{\infty}(2 l+1) \sin ^{2} \eta_{l}(k) \\
& \sigma_{M T}=\frac{4 \pi}{k^{2}} \sum_{l=0}^{\infty}(l+1) \sin ^{2}\left[\eta_{l}(k)-\eta_{l+1}(k)\right] \\
& \frac{d \sigma}{d \omega}=\frac{1}{k^{2}}\left|\sum_{l=0}^{\infty}(2 l+1) \exp \eta_{l} \sin \eta_{l}(k) P_{l}(\cos \theta)\right|^{2}
\end{aligned}
$$


where $\theta$ is the scattering angle and $P_{l}(x)$ are the Legendre polynomials.

Classically, one can use nonlinear least-square regression procedures in order to fit MERT to chosen experimental data and determine unknown parameters in the effective range expansions given by (2). However, due to a multiparameter nature of the model, it is more appropriate to use a Bayesian inference for parameter estimation [10]. In contrast to the classical fitting, the Bayesian inference does not provide single point estimation in parameter space but rather the probability density functions (PDFs) of model parameters that are shaped by observational data. Once a posteriori PDFs for each parameter are known, it is useful to provide a point estimation representing "best-fit" values together with an estimate of its errors. It can be done using either the mode or the mean value of PDF with the variance of distribution representing its uncertainty [10]. Alternatively, one can give a credible region representing predictive probability limit of the model due to parameters uncertainties (see ref. [9] for definition of this quantity). Bayesian parameter estimation requires the computation of multi-dimensional integrals and a good solution for this computational problem consists of implementing Markov chain Monte Carlo (MCMC) methods [10]. For our calculations, we adapt MCMC Matlab toolbox by Laine (http://helios.fmi.fi/ lainema/mcmc/) containing Delayed Rejection and Adaptive Metropolis (DRAM) sampling algorithm with multivariate Gaussian proposal distributions introduced by Haario et al. [21]. See ref. [9] for more details.

In all present calculations, the following theoretical value $\alpha=27.66 a_{0}^{3}$ of dipole static polarizability was used [22]. This value is consistent with other theoretical and experimental determinations [23]. Moreover, it was checked that outcome of MERT fits is very weakly sensitive to the changes of dipole polarizability within the range of $27-$ $-27.8 a_{0}^{3}$ where most of the literature data are reported. In addition, it was found (see next section) that the inclusion of the quadrupole polarizability $\beta=209.85 a_{0}^{5}$ [22] in Ali and Fraser expressions [20] for the scattering phase-shifts of higher partial waves $(l>2)$ is necessary in order to improve the agreement with more sophisticated theoretical calculations of $\eta_{l}$ at higher energies.

\section{Analysis}

\subsection{Total Cross Sections}

In spite of the fact that less measurements are available for Xe than for other noble gases, a pretty good agreement exists for TCS, see Fig. 1. In the region of the Ramsauer-Townsend (R-T) minimum, discrepancies in TCS

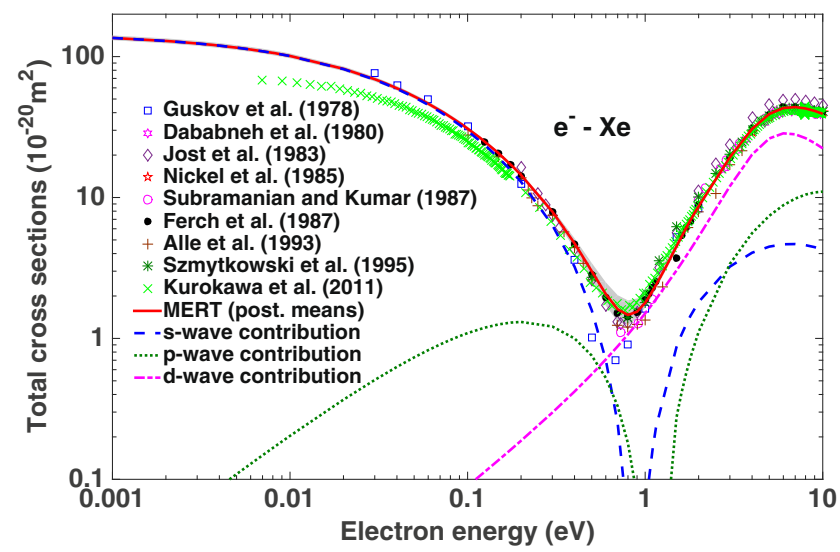

Fig. 1 a MCMC-MERT fit to total cross-sections by Ferch et al. [30]. The solid line is calculated using the posterior means of the parameters. The mean contributions of $s, p$, and $d$ partial waves are also shown. The grey area in the plot (clearly visible only near R-T minimum) corresponds to $50 \%$ credibility region due to uncertainties of MERT parameters. The fit is compared with experimental data by: Guskov et al. [25], Dababneh et al. [26], Jost et al. [27], Nickiel et al. [28], Subramanian et al. [29], Alle et al. [31], Szmytkowski et al. [32], and Kurokawa et al. [14, 15]

are smaller than for $\mathrm{Kr}$ (see extensive review of scattering cross-sections in ref. [24]). Moreover, all TCSs are measured in absolute way, and in low-energy range, they correspond to the elastic integral cross-sections. Therefore, the most accurate scattering phase-shifts can be derived by fitting MERT to available experimental TCSs. Benefiting from good agreement between experimental TCSs, the scattering phase shifts were first determined by performing simultaneous robust fit of (5) to large collection of TCS data sets (using MATLAB routine for nonlinear leastsquare regression of multiple data sets) similarly as it was done in analogous analysis for neon [6]. For present analysis, only those experimental TCSs were chosen that extend well below the energy corresponding to the R-T minimum: Guskov et al. [25], Jost et al. [27], Ferch et al. [30], Alle et al. [31], and Szmytkowski et al. [32]. The most recent experimental data of Kurokawa et al. [14, 15] has not been included in the analysis because these cross-sections are much lower in low energy limit than other experiments (see Fig. 1). Moreover, MERT fit to this dataset alone is not able to reproduce R-T minimum (i.e., the derived s-wave phase-shift does not change the sign in considered energy range).

In addition, to check the compatibility between available experimental TCSs, MTCSs, and DCSs measured in the region of R-T minimum in different laboratories with different techniques, the scattering phase-shifts were also derived via Bayesian predictive analysis. It has been done using Markov Chain Monte Carlo (MCMC) fitting methods separately applied to all single TCSs. Figure 1 presents an exemplary MCMC fit to TCS by Ferch et al. [30]. Both the 


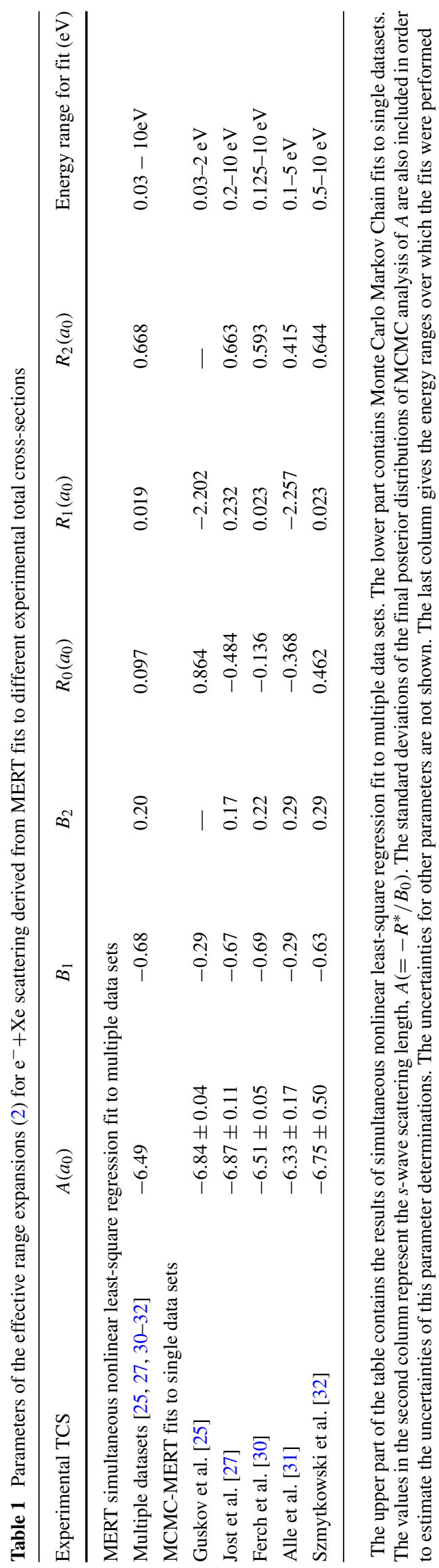

mean posterior curve (solid line) together with the region of $50 \%$ credibility (grey area) are illustrated. Parameters of the effective range expansions derived from simultaneous robust fit to several TCSs and from MCMC analysis of single datasets are given in Table 1.

It is clear from Table 1 that the $s$-wave scattering length (A) together with parameters $B_{1}$ and $B_{2}$ determined from MCMC analysis of TCS by Ferch et al. [30] are in the best agreement with the corresponding parameters derived from simultaneous fit to several TCSs. It implies that the related scattering phase-shifts calculated using these parameters also remain in the best accordance. These two MERT-derived phase-shift datasets are presented in Fig. 2 ( $\eta_{l}$ derived from other TCSs are not shown for clarity of figure) where they are compared with relativistic calculations of Sin Fai Lam [16], Sienkiewicz and Baylis [18], and McEachran and Stauffer [17]. The latter data were published only for high energies, $E>5 \mathrm{eV}$, therefore the non-relativistic calculations of the same authors [33] available at lower energies are also included for comparison. It has to be added that recently [34] the same authors reported the results of relativistic calculations with complex optical potential of elastic and momentum transfer cross sections for electron scattering from zero to $1 \mathrm{keV}$. They were able to obtain a consistent set of cross sections over this entire energy range. However, no information about energy dependence of scattering phase-shifts has been provided.

Both the $s$-wave phase shift (dotted line, Fig. 2) derived from a simultaneous fit to several considered TCSs and from MCMC analysis of TCS by Ferch et al. [30] (solid line, Fig. 2) are in good agrement with the data by Sienkiewicz and Baylis. The results of Sin Fai Lam and old data of McEachran and Stauffer [17, 33] lie systematically lower than the present estimates. On the other hand, MERTderived $p$-wave and $d$-wave phase shifts are generally in much better agreement with all theories. The present results lay more or less in-between relativistic calculations indicating that the spin-polarization effects for $p$ - and $d$-waves are noticeable in considered energy range $(E<10 \mathrm{eV})$ but still relatively small when compared to the dominant contribution from a adiabatic long-range dipole polarization interaction.

Figure 2 presents also the $f$-wave phase-shifts $\left(\eta_{3}\right)$ calculated using expressions introduced by Ali and Fraser [20] for scattering from pure long-range potentials (dipole and quadrupole polarizabilities). For comparison purposes, the calculations assuming non-zero quadrupole polarizability are shown together with computations neglecting this part of the long-range interaction. The latter corresponds to the Born approximation for the scattering phaseshifts induced by pure dipole polarization potential. It is clear that the quadrupole polarizability starts to play a 


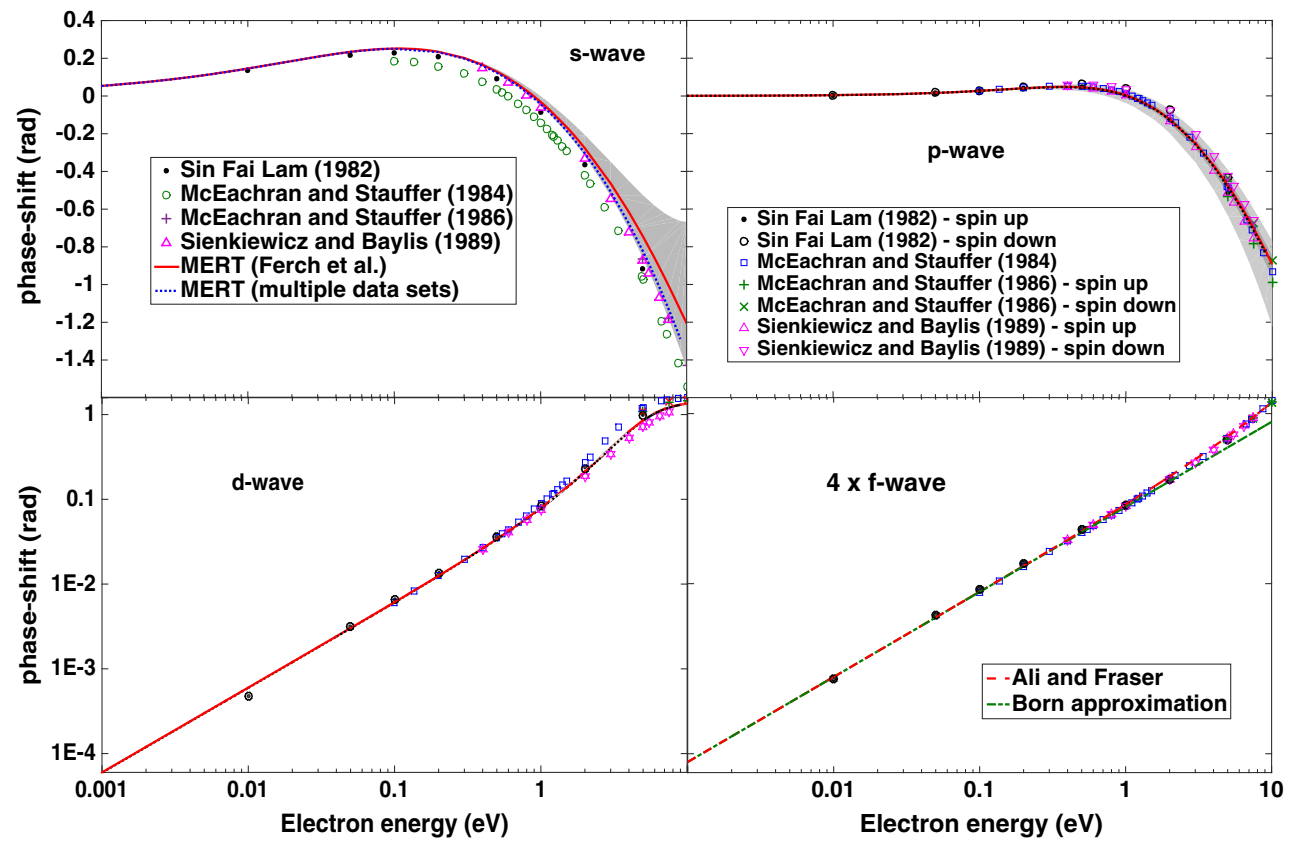

Fig. 2 MERT-derived $s$-, $p$-, and $d$-wave scattering phase shifts obtained from MCMC fits of the model to total cross-sections by Ferch et al. [30]. The grey area in the plot corresponds to $50 \%$ posterior region due to uncertainties of MERT parameters. The dotted line represents phase-shifts derived from simultaneous classical robust fit to several TCSs [27, 30-32]. The present results are compared with the

role at high energies, $E>1 \mathrm{eV}$, and thus it has to be included within the MERT framework if we want to describe scattering cross-sections up to the threshold for the first inelastic process. The good agreement observed (Fig. 2) between present results for $f$-wave and other theories shows that at low-energy range $(E<10 \mathrm{eV})$, for high $l$ numbers, the rigorous treatment of short-range effects (including relativistic ones) provides only minor corrections to the phase-shifts induced by pure long-range forces.

Comparing MERT-derived differential (DCS) and momentum transfer (MTCS) cross-sections with different experiments, it was found that the largest number of calculations of Sin Fai Lam [16], McEachran and Stauffer [17, 33], and Sienkiewicz and Baylis [18]. The $f$-wave phase shifts $\left(\eta_{3}\right)$ calculated using expressions introduced by Ali and Fraser [20] $(3,4)$ with and without (Born approximation) inclusion of quadrupole polarizability are also shown for comparison. The latter results are multiply by 4 in order to match the scale on the vertical axis of $d$-wave plot experimental points lay in the $50 \%$ credibility region determined from TCS by Ferch et al. [30]. Moreover, the MCMC analysis of this dataset gives the lowest uncertainty for the scattering length. Similarly, low uncertainty is seen in Guskov's data [25], but the energy range of their measurements (and of the fit in consequence) was much narrower than that by Ferch et al. [30]. Moreover, this data are lower in R-T minimum than other experimental TCSs. Furthermore, we do not know details of their experimental procedures and setup. On the other hand, experimental TCS of Ferch was determined using well-described time of flight (TOF) system purposely designed for very low-energy electron scattering [37]. In the subsequent subsections, only the
Table 2 Comparison of the $\mathrm{e}^{-}+$Xe scattering lengths obtained in previous studies

\begin{tabular}{ll}
\hline Source & $A\left(a_{0}\right)$ \\
\hline O’Malley [11] & $-6.9 \leq A \leq-5.6$ \\
MERT, Guskov et al. [25] & -6.8 \\
MERT, Weyhreter et al. [13] & -6.527 \\
MERT, Kurokawa et al. [14] & -5.13 \\
Swarm analysis, Hunter et al. [42] & -6.08 \\
Swarm analysis, Pack et al. [43] & -7.072 \\
Optical absorption, Rupnik et al. [38] & -5.83 \\
Theory, McEachran et al. [33] & -5.232 \\
Theory, Zatsarinny et al. [39] & -6.79 \\
\hline
\end{tabular}




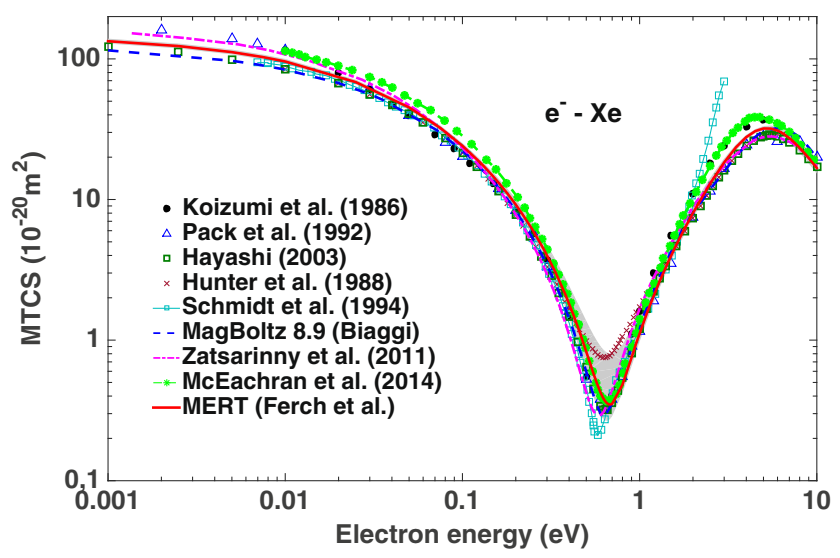

Fig. 3 MERT-derived momentum transfer cross-sections obtained from a MCMC fit of model to total cross-sections by Ferch et al. [30]. The grey area in the plot corresponds to $50 \%$ posterior regions due to uncertainties of MERT parameters. The present results are compared with the swarm-derived MTCS of , Koizumi et [41], Hunter et al. [42], Pack et al. [43], Schmidt et al. [44], and Hayashi [45]. The theoretical calculations by S.F. Biagi's FORTRAN code, MagBoltz 8.9 (http:// consult.cern.ch/writeup/magboltz), BSR quantum-mechanical calculations by Zatsarinny and Bartschat [39], and relativistic results of McEachran and Stauffer [34] are also shown for comparison

results related to this fit will be presented though the results obtained from MERT analysis of other datasets such as Jost et al. [27] and Szmytkowski et al. [32] are of similar quality (all stay within the range of combined errors of all available experimental MTCS and DCS).

On the basis of this comparative study, it is also concluded that the value of scattering length, $(-6.51 \pm 0.05) a_{0}$, derived from time of flight data by Ferch et al. [30] is the most probable. This result is in very good agreement with the value of $-6.527 a_{0}$ obtained using original MERT analysis applied to very-low-energy elastic DCSs by Weyhreter et al. [13]. Moreover, it is also consistent with $A=-6.5 a_{0}$ determined by O'Malley [11] from very old TCS by Ramsauer and Kollath [35]. The comparison of $\mathrm{e}^{-}+$Xe scattering lengths determined experimentally or theoretically by different methods is given in Table 2 . The relatively large negative $s$-wave scattering length obtained in the present and previous works supports the presence of virtual state enhancing the scattering cross-sections in the limit of zero energy [36].

\subsection{Momentum Transfer Cross Sections}

Swarm-derived momentum cross-sections are generally in good agreement in the region of Ramsauer-Townsend minimum [40]. Except determinations of Hunter et al. [42] and Schmidt et al. [44], other experimental results of Koizumi et al. [41], Pack et al. [43] and Hayashi [45] are consistent within few percent accuracy (see Fig. 3) giving R-T minimum of $(0.30-0.38) \cdot 10^{-20} \mathrm{~m}^{2}$ at $0.6-0.7 \mathrm{eV}$. Most of the recent theoretical calculations (http://consult.cern. ch/writeup/magboltz) [34, 39] confirm these experimental determinations. Present MERT analysis also reproduces very well experimental results, see Fig. 3, giving a minimum of $0.35 \cdot 10^{-20} \mathrm{~m}^{2}$ at $0.68 \mathrm{eV}$.

\subsection{Differential Cross Sections}

The differential cross-sections (DCSs) calculated with the MERT-derived phase shifts are compared with available

Fig. 4 Angular dependencies of MERT-derived differential cross-sections obtained from a MCMC fit of model to total cross-sections by Ferch et al. [30]. The present results are compared at $1,5,7.9$, and $10 \mathrm{eV}$ with the experimental DCS of Register et al. [46], Weyhreter et al. [13], Gibson et al. [47], and Linert et al. [48]

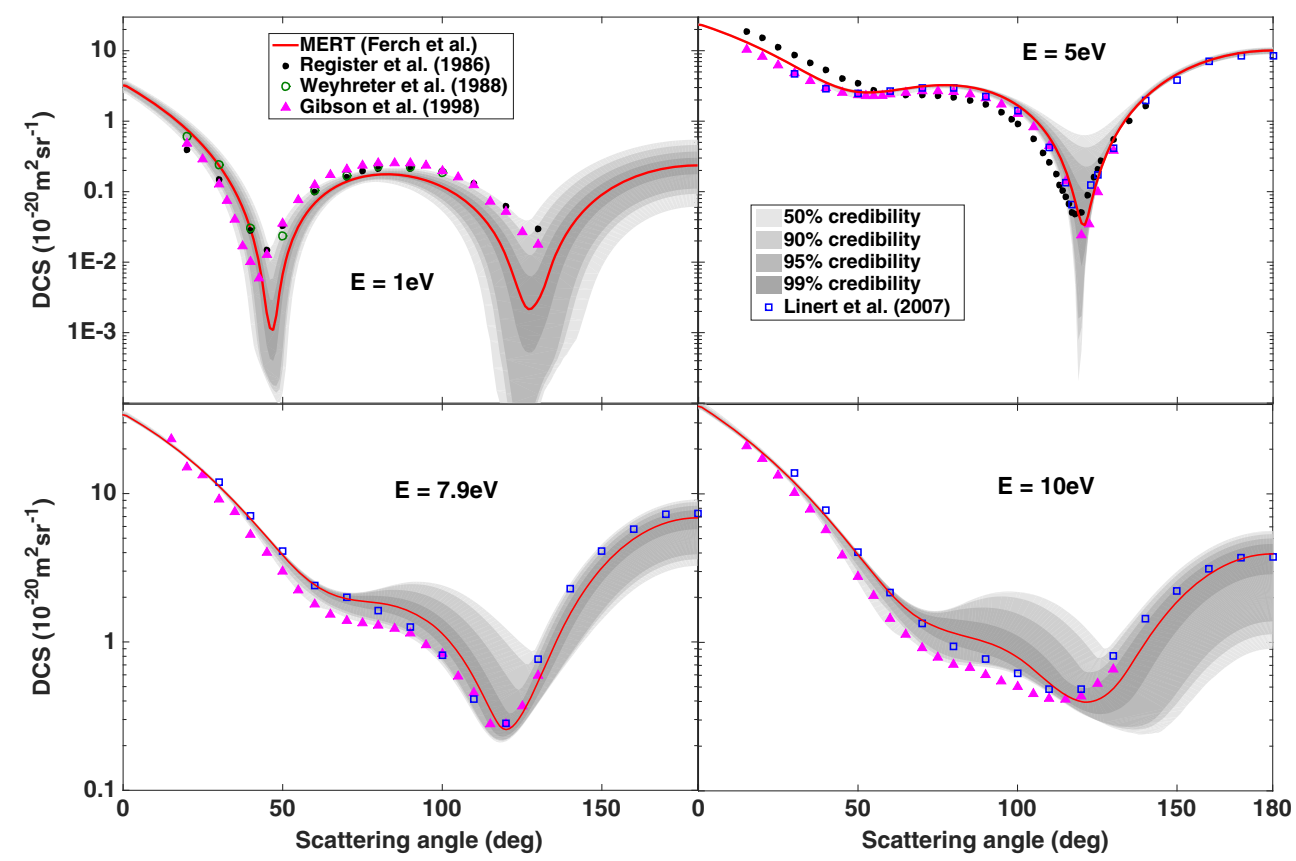




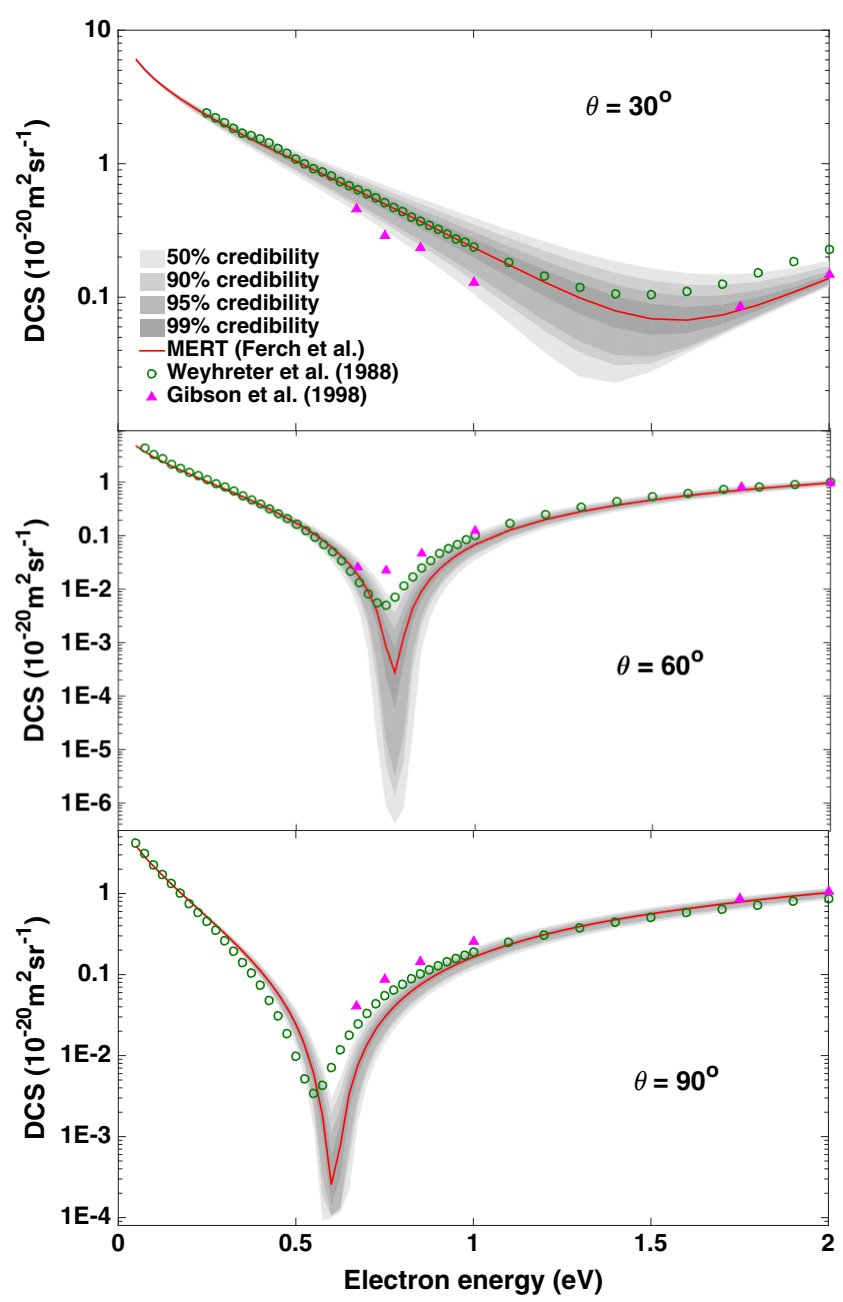

Fig. 5 Energy dependencies of MERT-derived differential crosssections obtained from MCMC fit of model to total cross-sections by Ferch et al. [30]. The present results are compared at 30, 60, and $90^{\circ}$ with the experimental DCS of Weyhreter et al. [13] and Gibson et al. [47]

experimental DCS data [13, 46-48] in Figs. 4 and 5. The agreement has to be judged as good, being at least at the same level of consistency as more advanced calculations (see for example refs. [47, 48] where the extensive comparison between experiments and theoretical calculations is done). In particular, MERT is in quite good accordance with high-angle-data measured by Linert et al. [48] with magnetic angler changer in hardly accessible angular region $\left(>150^{\circ}\right)$. Surprisingly, a good agreement is seen up to $10 \mathrm{eV}$.

\section{Scattering Length for Noble Gases}

This article completes our picture of MERT applicability to noble gases [5-7]. Figure 6 shows an attempt to correlate the $s$-wave scattering lengths $(A)$ for all noble gases

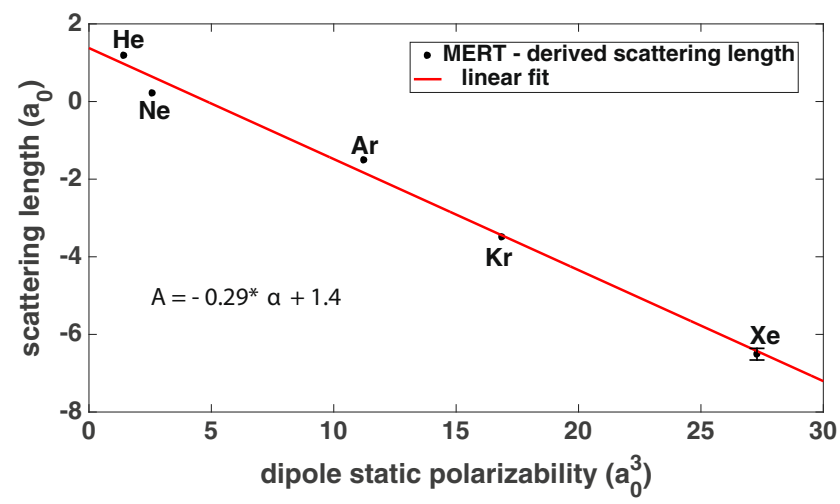

Fig. 6 MERT-derived $s$-wave scattering lengths (dots) for rare gases [5-7] plotted versus static dipole polarizability. The present result for Xenon is given with $3 \sigma$ error bar determined from Bayesian predictive analysis. The linear fit (solid line) is presented to show a general tendency in $A(\alpha)$ dependency

versus static dipole polarizability $(\alpha)$ as hypothesized by Reisfeld and Asaf [49]. The values of the scattering length, except the present result for Xenon, were determined in our previous MERT studies [5-7] using "traditional" fitting techniques. Figure 6 presents also the linear fit to $A(\alpha)$ in order to show a general tendency in the relation between these two quantities. The similar dependency has been already predicted by Reisfeld and Asaf [49] who showed that $A(\alpha)$ can be indeed approximately expressed by the linear relationship if the electron-target interaction potential is approximated by the short-range hard-core repulsive part plus the long-range dipole polarization potential. Our MERT-computed scattering-lengths seem to partially confirm these results though the parametric dependence on the size of the hard core does not seem to be so crucial as it is postulated by the expression derived by Reisfeld and Asaf (see Eq. 6 in ref. [49].

In most cases, the scattering lengths for noble gases have been determined by extrapolating experimental or theoretical results using modified effective range theory. More fundamental ( $a b$ initio) calculations are hardly ever carried out in very low (near-to-zero) energy region due to the tedious nature of computations. Moreover, the direct experimental determinations of $A$ are typically subject to large errors. The advantage of present Markov Chain Monte Carlo effective range analysis is the ability to predict error bars for the theoretical estimates of model parameters when compared to the traditional fitting methods. We plan to apply Bayesian inference for other noble gases: helium, neon, argon, and krypton, in order to estimate the uncertainties of scattering lengths that were determined in our previous "traditional" MERT studies [5-7]. We expect that the inclusion of error bars for extrapolated values can explain the small deviations from linear dependency of $A(\alpha)$. 


\section{Conclusions}

This work shows that non-relativistic spinless model such as modified effective range theory (MERT) is able to describe cross-sections for low-energy electron $(E<10 \mathrm{eV})$ elastic scattering from Xenon atom at the same level of consistency with experiments as more advanced theoretical approaches. This indicates that a long-range adiabatic dipole polarization potential $\left(\sim r^{-4}\right)$ is a dominant contribution to the overall $\mathrm{e}^{-}+\mathrm{Xe}$ interaction in considered energy range. The spin polarization effects in scattering events, important for few lowest partial waves $l>0$, can be considered only as small corrections to non-relativistic case.

Bayesian predictive analysis of experimental total crosssections (TCS) in the region of Ramsauer-Townsend minimum shows that the time of flight data by Ferch et al. [30] stays in the best consistency with momentum transfer and differential cross-sections measured in different laboratories at the same energy range. As the result, the value of the scattering length, $A=(-6.51 \pm 0.05) a_{0}$, predicted by Bayesian inference technique from TCS of Ferch et al. [30] is recommended in this work.

In final, this paper completes the picture of MERT applicability for all noble gases [5-7] showing that the present model can be used to describe the scattering cross-sections from zero-energy up to the threshold for the first inelastic process for $\mathrm{He}, \mathrm{Ne}, \mathrm{Ar}, \mathrm{Kr}$, and $\mathrm{Xe}$. Moreover, in our earlier works, [5, 8] we showed that MERT is also applicable in relatively wide energy range for simple (almost spherical) molecules such as $\mathrm{H}_{2}$ and $\mathrm{CH}_{4}$. The present model could be also potentially used to describe cross-sections for electron scattering from targets of general symmetry at very lowimpact energies when de Broglie wavelength of the electron is much greater than the dimensions of the molecule. In such a case, the anisotropy in electron-non-spherical target interaction can be neglected preserving the validity of the effective range theory. This work is in progress.

Acknowledgments This work is supported by the grant 2014/15/D/ST2/02358 of National Science Center in Poland. The author would like to thank Prof. G Karwasz for fruitful discussions.

Open Access This article is distributed under the terms of the Creative Commons Attribution 4.0 International License (http:// creativecommons.org/licenses/by/4.0/), which permits unrestricted use, distribution, and reproduction in any medium, provided you give appropriate credit to the original author(s) and the source, provide a link to the Creative Commons license, and indicate if changes were made.

\section{References}

1. T.F. O’Malley, L. Spruch, L. Rosenberg, J. Math. Phys. 2, 491 (1961)
2. S.J. Buckman, J. Mitroy, J. Phys, B: At. Mol. Phys. 22, 1365 (1989)

3. Z. Idziaszek, G. Karwasz, Phys. Rev. A 73, 064701 (2006)

4. Z. Idziaszek, G. Karwasz, Eur. Phys. J. D. 51, 247 (2009)

5. K. Fedus, G.P. Karwasz, Z. Idziaszek, Phys. Rev. A 88, 012704 (2013)

6. K. Fedus, Braz. J. Phys. 44, 622 (2014)

7. K. Fedus, Phys. Scr. 89, 105401 (2014)

8. K. Fedus, G.P. Karwasz, Eur. Phys. J. D 68, 93 (2014)

9. K. Fedus, J. Franz, G. Karwasz, Phys. Rev. A. 91, 062701 (2015)

10. P. Gregory, Bayesian Logical Data Analysis for the Physical Sciences (Cambridge University Press, New York, 2005)

11. T.F. O’Maley, Phys. Rev. 13, 1020 (1963)

12. Y.K. Guskov, R.V. Savvov, V.A. Slobodyanyuk, Sov. Phys. Tech. Phys. 23, 167 (1978)

13. M. Weyhreter, B. Barzick, A. Mann, F. Linder, Z. Phys. D. 7, 333 (1988)

14. M. Kurokawa, M. Kitajima, K. Toyoshima, T. Kishino, T. Odagiri, H. Kato, M. Hoshino, H. Tanaka, K. Ito, Phys. Rev. A 84, 062717 (2011)

15. M. Kitajima, M. Kurokawa, T. Kishino, K. Toyoshima, T. Odagiri, H. Kato, K. Anzai, M. Hoshino, H. Tanaka, K. Ito, Eur. Phys. J. D 66, 130 (2012)

16. L.T. Sin Fai Lam, J. Phys. B: At. Mol. Phys. 15, 119 (1982)

17. R.P. McEachran, A.P. Stauffer, J. Phys. B: At. Mol. Phys. 19, 3523 (1986)

18. J.E. Sienkiewicz, W.E. Baylis, J. Phys. B: At. Mol. Phys. 22, 3733 (1989)

19. R.P. McEachran, A.P. Stauffer, Eur. Phys. J. D 68, 153 (2014)

20. M.K. Ali, P.A. Fraser, J. Phys. B: At. Mol. Phys. 10, 3091 (1977)

21. H. Haario, M. Laine, A. Mira, E. Saksman, Stat. Comput. 16, 339 (2006)

22. A. Nicklass, M. Dolg, H. Stoll, H. Preuss, J. Chem. Phys. 102, 22 (1995)

23. T.N. Olney, N.M. Cann, G. Cooper, C.E. Brion, Chem. Phys. 223, 59 (1997)

24. A. Zecca, R.S. Brusa, G.P. Karwasz, No 19, 3 (1996)

25. Y. Guskov, R.V. Savvov, V.A. Slobodyanyuk, Sov. Phys. Tech. Phys. 23, 167 (1978)

26. M.S. Dababneh, W.E. Kauppila, J.P. Downing, F. Laperriiere, V. Pol, J.H. Smart, T.S. Stein, Phys. Rev. A 22, 1872 (1980)

27. K. Jost, P.G.F. Bisling, F. Eschen, M. Felsmann, L. Walther, XIII International Conference on Physics of Electronic and Atomic Collisions, Berlin, ed. by J. Eichler, et al. Abstract p. 91, (North-Holland, Amsterdam, 1983)

28. J.K. Nickel, K. Imre, D.F. Register, S. Trajmar, J. Phys. B: At. Mol. Phys. 18, 125 (1985)

29. K.P. Subramanian, V. Kumar, J. Phys. B: At. Mol. Phys. 20, 5505 (1987)

30. J. Ferch, F. Simon, G. Strakeljahn, XV International Conference on Physics of Electronic and Atomic Collisions, Brighton, ed. by J. Geddes, et al. Abstract p. 132, (North-Holland, Amsterdam, 1987)

31. D.T. Alle, M.J. Brennan, S.J. Buckman, XVIII International Conference on Physics of Electronic and Atomic Collisions, Aarhus, ed. by T. Andersen, et al. Abstract p. 127 (Aarhus University, 1993)

32. Cz. Szmytkowski, K. Macig, G. Karwasz, Phys. Scr. 54, 271 (1996)

33. R.P. McEachran, A.P. Stauffer, J. Phys. B: At. Mol. Phys. 17, 2507 (1984)

34. R.P. McEachran, A.D. Stauffer, Eur. Phys. J. D 68, 153 (2014)

35. C. Ramsauer, R. Kollath, Ann. Physik. 12, 837 (1932) 
36. D. Field, N.C. Lunt, Jones S.L., J.-P. Ziesel, Phy. Rev. A 64, 022708 (2001)

37. J. Ferch, B. Granitza, C. Masche, W. Raith, J. Phys, B: At. Mol. Phys. 18, 967 (1985)

38. K. Rupnik, U. Asaf, S.P. McGlynn, J. Chem. Phys 92, 2303 (1990)

39. O. Zatsarinny, K. Bartschat, J. Phys. B: At. Mol. Opt. Phys. 37, 4693 (2004). http://fr.lxcat.net-dataforXe

40. M.C. Bordage, S.F. Biagi, L.L. Alves, K. Bartschat, S. Chowdhury, L.C. Pitchford, G.J.M. Hagelaar, W.L. Morgan, V. Puech, O. Zatsarinny, J. Phys. D: Appl. Phys. 46, 334003 (2013)

41. T. Koizumi, E. Shirakawa, I. Ogawa, J. Phys. B: At. Mol. Phys. 19, 2331 (1986)

42. S.R. Hunter, J.G. Carter, L.G. Christophorou. Phys. Rev. A 38, 5539 (1988)
43. J.L. Pack, R.E. Voshall, A.V. Phelps, L.E. Kline, J. Appl. Phys. 71, 5363 (1992)

44. B. Schmidt, K. Berkhan, B. Götz, M. Müller, Phys. Scr. 53, 30 (1994)

45. M. Hayashi, Report NIFS-DATA-79, Japan National, Institute for Fusion Science (2003)

46. D.F. Register, L. Vuskovic, S. Trajmar, J. Phys. B: At. Mol. Phys. 19, $1685(1986)$

47. J.C. Gibson, D.R. Lun, L.J. Allen, R.P. McEachran, L.A. Parcell, S.J. Buckman, J. Phys. B: At. Mol. Phys. 31, 3949 (1998)

48. I. Linert, B. Mielewska, G.C. King, M. Zubek, Phys. Rev. A 76, 032715 (2007)

49. G. Reisfeld, U. Asaf, Phys. Rev. A 49, 348 (1994) 